\title{
Polypectomy as a diagnostic tool for ileocecal lymphoma
}

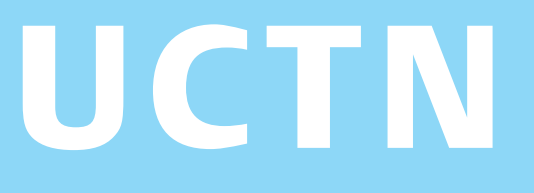

A 59-year-old man underwent a surveillance colonoscopy after endoscopic resection of multiple polyps. Colonoscopy showed a reddish and swollen ileocecal valve. Although the colonoscopy could not be introduced into the terminal ileum, the patient was asymptomatic. The surface of the swollen ileocecal valve was reddish, rather irregularly shaped, and nodular-like, mimicking a lump consisting of multiple swollen lymphoid follicles (Figure 1a). Small ulcers were also partially evident on the surface, and the mass was demonstrated to be elastichard with biopsy forceps (Figure $\mathbf{1} \mathbf{b}$ ). Multiple endoscopic biopsy samples were taken from different areas, including the small ulcers, for histological evaluation, but no definite diagnosis could be obtained. As a diagnosis could not be established by repeated biopsies, polypectomy was performed to remove a larger specimen for histological evaluation. The resected specimen was sliced into two pieces, and macroscopic observation revealed that it consisted of round, whitish nodules resembling lymph nodes (Figure $\mathbf{2}$ a,b). Histologically, the features suggested malignant lymphoma. The patient underwent surgical resection, and the final diagnosis was diffuse large B-celltype malignant lymphoma.

The ileocecal area and ileum are the regions most frequently affected by primary small- and large-intestinal non-Hodgkin's lymphoma, and most of such cases, like the present one, are diffuse large B-cell lymphoma [1]. According to the American Society for Gastrointestinal Endoscopy (ASGE) guidelines, during colonoscopy, every effort should be made to obtain a tissue diagnosis when encountering polyps, mass lesions, or colonic strictures [2]. Unfortunately, in the present case, a correct diagnosis could not be established from multiple biopsy samples. As definitive diagnosis could only be made from large tissue fragments taken from representative portions of the lesion, polypectomy was performed and a diagnosis was established successfully. In other cases,

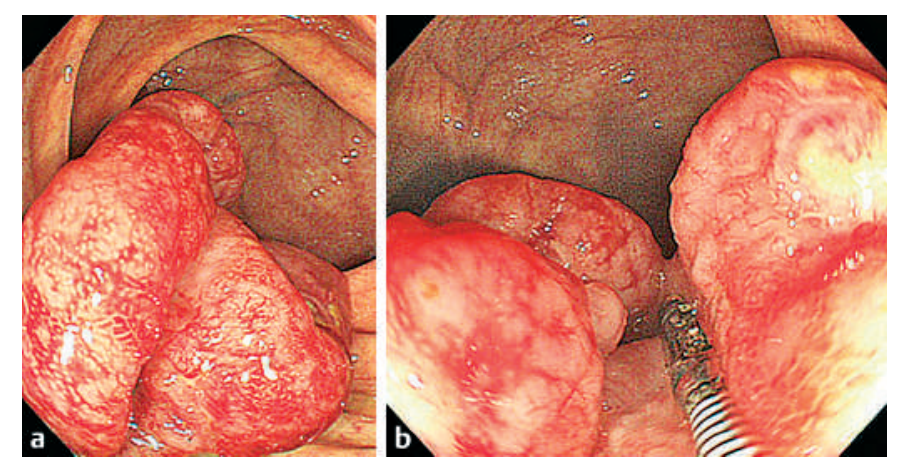

Figure 1 a Colonoscopy showed a reddish and swollen ileocecal valve. b The surface of the swollen ileocecal valve was shaped rather irregularly with small ulcers in part, and with a nodular-like mass consisting of multiple swollen lymphoid follicles.
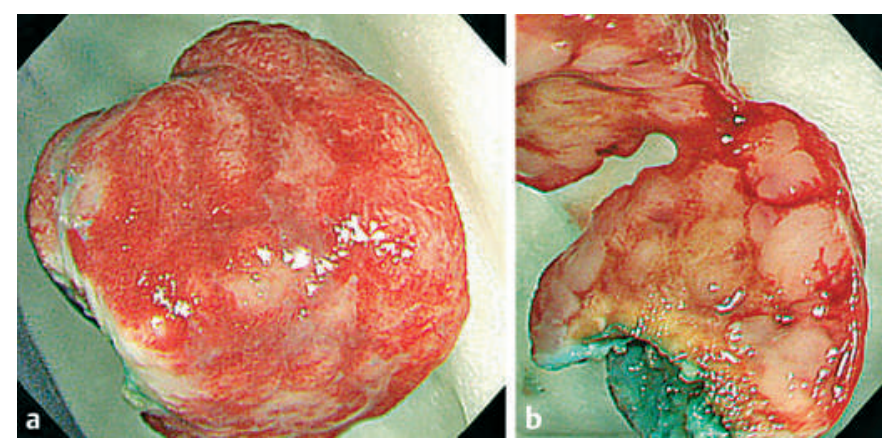

Figure 2 a Endoscopic view of the specimen resected by polypectomy. b The resected specimen was sliced into two pieces and this revealed that the mass consisted of round, whitish nodules resembling lymph nodes.

endoscopic ultrasound-guided fine-needle aspiration biopsy may provide an accurate diagnosis, particularly in patients for whom previous endoscopic forceps biopsy has been unsuccessful [3]. However, this procedure is not always available, as special equipment and skill are needed.

\section{Endoscopy_UCTN_Code_CCL_1AC_2AC}

\section{H. Nakamura', K. I. Fu², J. Matsumoto ${ }^{3}$,}

\section{Y. Kaji ${ }^{2}$, T. Fujimori ${ }^{4}$}

${ }^{1}$ Department of Gastroenterology, Chofu Surgical Clinic, Tokyo, Japan

2 Department of Radiology, Dokkyo University School of Medicine, Tochigi, Japan

${ }^{3}$ Department of Surgery, Tokyo Metropolitan Fuchu Hospital, Tokyo, Japan

${ }^{4}$ Department of Surgical and Molecular Pathology, Dokkyo University School of Medicine, Tochigi, Japan.
References

${ }^{1}$ Kohno S, Ohshima K, Yoneda S et al. Clinicopathological analysis of 143 primary malignant lymphomas in the small and large intestines based on the new WHO classification. Histopathology 2003; 43: 135-143

2 Davila RE, Rajan E, Adler D et al. ASGE guideline: the role of endoscopy in the diagnosis, staging, and management of colorectal cancer. Gastrointest Endosc 2005; 61: 1 - 7

${ }^{3}$ Vander Noot MR 3rd, Eloubeidi MA, Chen VK et al. Diagnosis of gastrointestinal tract lesions by endoscopic ultrasound-guided fine-needle aspiration biopsy. Cancer 2004; 102: $157-163$

\section{Corresponding author}

\section{K. I. Fu, MD, PhD}

Department of Radiology

Dokkyo University School of Medicine . 880 Kitakobayashi · Mibu, Shimotuga ·

Tochigi, 321-0293 - Japan

Fax: $\quad+81-282-86-5678$

Email: fukuangi@hotmail.com 